PROCEEDINGS OF THE

AMERICAN MATHEMATICAL SOCIETY

Volume 131, Number 8, Pages 2459-2465

S 0002-9939(03)06947-8

Article electronically published on January 8, 2003

\title{
GRAPHS THAT ARE NOT COMPLETE PLURIPOLAR
}

\author{
ARMEN EDIGARIAN AND JAN WIEGERINCK
}

(Communicated by Mei-Chi Shaw)

\begin{abstract}
Let $D_{1} \subset D_{2}$ be domains in $\mathbb{C}$. Under very mild conditions on $D_{2}$ we show that there exist holomorphic functions $f$, defined on $D_{1}$ with the property that $f$ is nowhere extendible across $\partial D_{1}$, while the graph of $f$ over $D_{1}$ is not complete pluripolar in $D_{2} \times \mathbb{C}$. This refutes a conjecture of Levenberg, Martin and Poletsky (1992).
\end{abstract}

\section{INTRODUCTION}

Levenberg, Martin and Poletsky [6] have conjectured that if $f$ is a holomorphic function, which is defined on its maximal domain of existence $D \subset \mathbb{C}$, then the graph

$$
\Gamma_{f}=\{(z, f(z)): z \in D\}
$$

of $f$ over $D$ is a complete pluripolar subset of $\mathbb{C}^{2}$. I.e., there exists a plurisubharmonic function on $\mathbb{C}^{2}$ such that it equals $-\infty$ precisely on $\Gamma_{f}$ (see e.g. 4]). They gave support for this conjecture in the sense that they could prove it for some lacunary series. More support was provided by Levenberg and Poletsky [7] and by the second author [9, 10, 11]. Nevertheless, in this paper we show that the conjecture is false.

In fact we have

Theorem 1.1. Let $D_{1} \subset D_{2}$ be domains in $\mathbb{C}$. Assume that $D_{2} \backslash D_{1}$ has a density point in $D_{2}$. Then there exists a holomorphic function $f$ with domain of existence $D_{1}$ such that the graph $\Gamma_{f}$ of $f$ over $D_{1}$ is not complete pluripolar in $D_{2} \times \mathbb{C}$.

In case $D_{2} \backslash D_{1}$ has no density point in $D_{2}$, it is known that $\Gamma_{f}$ is complete pluripolar in $D_{2} \times \mathbb{C}($ see $[10])$. If we take in Theorem 1.1 for $D_{1}$ the unit disc $\mathbb{D}$ and for $D_{2}$ the whole plane $\mathbb{C}$, we obtain the following corollary.

Corollary 1.2. There exists a holomorphic function $f$ defined on $\mathbb{D}$, which does not extend holomorphically across $\partial \mathbb{D}$, such that $\Gamma_{f}$ is not complete pluripolar in $\mathbb{C}^{2}$.

Received by the editors March 15, 2002.

2000 Mathematics Subject Classification. Primary 32U30; Secondary 31A15.

Key words and phrases. Plurisubharmonic function, pluripolar hull, complete pluripolar set, harmonic measure.

The first author was supported in part by KBN grant No. 5 P03A 03321 . The first author is a fellow of the A. Krzyżanowski Foundation (Jagiellonian University). 
Theorem 3.2 then states that such a function can even be smooth up to the boundary of $\mathbb{D}$.

The first-named author thanks Marek Jarnicki, Witold Jarnicki, and Peter Pflug for very helpful discussions.

\section{Graphs With NON-TRIVIAL PLURIPOlar HULL}

The pluripolar hull of a pluripolar set $K \subset \Omega$ is the set

$$
K_{\Omega}^{*}=\left\{z \in \Omega:\left.u\right|_{K}=-\infty, u \in \operatorname{PSH}(\Omega) \Longrightarrow u(z)=-\infty\right\} .
$$

By $\omega(z, E, D)$ we denote as usual the harmonic measure of a subset $E$ of the boundary of a domain $D$ in $\mathbb{C}$ at the point $z$ in $D$ (see e.g. [8]).

Let $A=\left\{a_{n}\right\}_{n=1}^{\infty}$ be a countable dense subset of $\partial D_{1}$. Under the assumptions of Theorem [1.1, there exists an $a \in\left(\partial D_{1}\right) \cap D_{2}$ such that $a \in \overline{A \backslash\{a\}}$. We may assume that $a \notin A$. Our function $f$ will be of the form

$$
f(z)=\sum_{j=1}^{\infty} \frac{c_{j}}{z-a_{j}} .
$$

We will choose $c_{n}$ very rapidly decreasing to 0 . In particular,

$$
\sum_{j=1}^{\infty}\left|c_{j}\right|<+\infty
$$

so the limit in (2.1) exists and is a holomorphic function on $D_{1}$.

Moreover, we will choose $c_{n}$ such that $\sum_{n=1}^{\infty} \frac{\left|c_{j}\right|}{\left|a-a_{j}\right|}<+\infty$. Hence, the series (2.1) will converge at $z=a$. We will denote its limit by $f(a)$. We will prove a version of Theorem 1.1 that elaborates on (2.1). However, no statement about extendibility is made at this point.

Theorem 2.1. Let $D_{1} \subset D_{2}$ be domains in $\mathbb{C}$, such that $D_{2} \backslash D_{1}$ has a density point in $D_{2}$. There exists a sequence $\left\{R_{n}\right\}_{n=1}^{\infty}$ of positive numbers such that for any sequence of complex numbers $\left\{c_{n}\right\}_{n=1}^{\infty}$ with $\left|c_{n}\right| \leq R_{n}$ we have $(a, f(a)) \in\left(\Gamma_{f}\right)_{D_{2} \times \mathbb{C}}^{*}$, where $f$ is given by (2.1). Here $\Gamma_{f}$ is the graph of $f$ over $D_{1}$.

Proof. We may assume that $a=0$. For $b \in \mathbb{C}$ and $r>0$ we set $\mathbb{D}(b, r)=\{z \in \mathbb{C}$ : $|z-b|<r\}, \mathbb{D}_{r}=\mathbb{D}(0, r)$ and $\mathbb{D}=\mathbb{D}_{1}$, the unit disc. Put

$$
\pi_{n}^{k}(z)=e^{\frac{2 \pi k i}{n}} z, \quad z \in \mathbb{C}, \quad k, n \in \mathbb{N}
$$

and

$$
B=\bigcup_{n=1}^{\infty} \bigcup_{k=1}^{n} \pi_{n}^{k}(A) .
$$

Note that $0 \notin B$ and that $B$ is also countable (and therefore thin at 0 ). By Corollary 4.8.3 in [4, there exists an open set $U \supset B$ such that $U$ is thin at 0 .

Step 1. We construct a sequence of radii $\left\{\rho_{n}\right\}_{n=1}^{\infty}$ with special properties, the main one being that $\bigcup_{n} \bigcup_{k=1}^{n} \pi_{n}^{k}\left(\mathbb{D}\left(a_{n}, \rho_{n}\right)\right)$ is thin at 0 .

It is a corollary of Wiener's criterion (see [8], Theorem 5.4.2) that there exists a sequence $r_{n} \rightarrow 0$ such that

$$
\partial \mathbb{D}_{r_{n}} \cap U=\varnothing, \quad n \in \mathbb{N} .
$$


Since $U$ is thin at 0 , there exists a subharmonic function $u$ on $\mathbb{C}$ such that

$$
\limsup _{U \ni z \rightarrow 0} u(z)=-\infty<u(0)
$$

(see e.g. Proposition 4.8.2 in [4]). Moreover, by scaling and adding a constant, we can assume that $u(0)=-\frac{1}{2}$ and $u<0$ on $\mathbb{D}$. By (2.3) there exists a $\rho>0$ such that $\overline{\mathbb{D}}_{\rho} \subset D_{2}, \partial \mathbb{D}_{\rho} \cap D_{1} \neq \varnothing, \partial \mathbb{D}_{\rho} \cap U=\varnothing$, and $u \leq-1$ on $U \cap \mathbb{D}_{\rho}$ (take $\rho=r_{n}$ with sufficiently big $n$ ).

Let $J \subset \partial \mathbb{D}_{\rho} \cap D_{1}$ be a closed arc. We can assume that

$$
J=\left\{e^{i \theta} \rho: \frac{2 \pi k_{0}}{n_{0}} \leq \theta \leq \frac{2 \pi\left(k_{0}+1\right)}{n_{0}}\right\}
$$

for some $k_{0}, n_{0} \in \mathbb{N}$.

Now we choose a sequence of positive numbers $\rho_{n} \in(0,1), n \in \mathbb{N}$, in the following way:

(1) Let $0<\rho_{1}<1$ be such that

(a) $\bigcup_{k=1}^{n_{0}} \pi_{n_{0}}^{k}\left(\mathbb{D}\left(a_{1}, \rho_{1}\right)\right) \subset U$;

(b) $\mathbb{D}_{\rho} \backslash \bigcup_{k=1}^{n_{0}} \pi_{n_{0}}^{k}\left(\overline{\mathbb{D}}\left(a_{1}, \frac{\rho_{1}}{2}\right)\right)$ is connected.

(2) Assume that $\rho_{1}, \ldots, \rho_{n-1}$ are chosen. Choose $0<\rho_{n}<1$ such that

(a) $\bigcup_{k=1}^{n_{0}} \pi_{n_{0}}^{k}\left(\mathbb{D}\left(a_{n}, \rho_{n}\right)\right) \subset U$;

(b) $\mathbb{D}_{\rho} \backslash \bigcup_{j=1}^{n} \bigcup_{k=1}^{n_{0}} \pi_{n_{0}}^{k}\left(\overline{\mathbb{D}}\left(a_{j}, \frac{\rho_{j}}{2}\right)\right)$ is connected.

Put $Y_{n}=\bigcup_{j=1}^{n} \bigcup_{k=1}^{n_{0}} \pi_{n_{0}}^{k}\left(\overline{\mathbb{D}}\left(a_{j}, \frac{\rho_{j}}{2}\right)\right)$. So, $Y_{n} \subset U$ is a closed set such that $\mathbb{D}_{\rho} \backslash Y_{n}$ is a domain and $\partial \mathbb{D}_{\rho} \cap Y_{n}=\varnothing$ for any $n \in \mathbb{N}$.

Step 2. We want to show that

$$
\omega\left(0, \partial \mathbb{D}_{\rho}, \mathbb{D}_{\rho} \backslash Y_{n}\right) \geq \frac{1}{2}, \quad n \in \mathbb{N} .
$$

Fix $n \in \mathbb{N}$. Put $v_{n}(z)=-\omega\left(z, \partial \mathbb{D}_{\rho}, \mathbb{D}_{\rho} \backslash Y_{n}\right)+u(z)$. It suffices to show that

$$
v_{n} \leq-1 \quad \text { on } \mathbb{D}_{\rho} \backslash Y_{n} .
$$

Observe that $-\omega\left(\cdot, \partial \mathbb{D}_{\rho}, \mathbb{D}_{\rho} \backslash Y_{n}\right) \leq 0$ and $u(\cdot) \leq 0$ on $\mathbb{D}_{\rho} \backslash Y_{n}$. Moreover, we have $\lim \sup _{z \rightarrow \partial \mathbb{D}_{\rho}}-\omega\left(z, \partial \mathbb{D}_{\rho}, \mathbb{D}_{\rho} \backslash Y_{n}\right) \leq-1$ and $\lim \sup _{z \rightarrow Y_{n}} u(z) \leq-1$. So, from the maximum principle for the subharmonic function $v_{n}$ we get (2.5) and, therefore, (2.4).

Step 3 . Here we want show that

$$
\omega\left(0, J, \mathbb{D}_{\rho} \backslash \bigcup_{j=1}^{n} \overline{\mathbb{D}}\left(a_{j}, \frac{\rho_{j}}{2}\right)\right) \geq \frac{1}{2 n_{0}}, \quad n \in \mathbb{N} .
$$

Put

$$
w_{n}(z)=\omega\left(z, \partial \mathbb{D}_{\rho}, \mathbb{D}_{\rho} \backslash Y_{n}\right)-\sum_{k=1}^{n_{0}} \omega\left(z, \pi_{n_{0}}^{k}(J), \mathbb{D}_{\rho} \backslash Y_{n}\right) .
$$

Note that $\bigcup_{k=1}^{n_{0}} \pi_{n_{0}}^{k}(J)=\partial \mathbb{D}_{\rho}$. Again from the maximum principle we obtain that $w_{n} \leq 0$ on $\mathbb{D}_{\rho} \backslash Y_{n}, n \in \mathbb{N}$.

Because $\pi_{n_{0}}^{k}\left(\mathbb{D}_{\rho} \backslash Y_{n}\right)=\mathbb{D}_{\rho} \backslash Y_{n}$, for any $k, n \in \mathbb{N}$, we find

$$
\omega\left(0, \pi_{n_{0}}^{k}(J), \mathbb{D}_{\rho} \backslash Y_{n}\right)=\omega\left(0, J, \mathbb{D}_{\rho} \backslash Y_{n}\right), \quad k \in \mathbb{N} .
$$


Hence,

$$
\omega\left(0, J, \mathbb{D}_{\rho} \backslash \bigcup_{j=1}^{n} \overline{\mathbb{D}}\left(a_{j}, \frac{\rho_{j}}{2}\right)\right) \geq \omega\left(0, J, \mathbb{D}_{\rho} \backslash Y_{n}\right) \geq \frac{1}{2 n_{0}}, \quad n \in \mathbb{N} .
$$

Step 4. Let $\left\{R_{n}\right\}_{n=1}^{\infty}$ be a sequence of positive numbers such that $C_{1}:=$ $\sum_{n=1}^{\infty} \frac{R_{n}}{\rho_{n}}<+\infty$ and, therefore, $\sum_{n=1}^{\infty} R_{n}<C_{1}$ (take e.g. $R_{n}=\frac{\rho_{n}}{n^{2}}$ ). Consider any sequence of complex numbers $\left\{c_{n}\right\}_{n=1}^{\infty}$ with $\left|c_{n}\right| \leq R_{n}$ and let $f$ be defined by (2.1).

Put

$$
f_{n}(z)=\sum_{j=1}^{n} \frac{c_{j}}{z-a_{j}}-\sum_{j=n+1}^{\infty} \frac{c_{j}}{a_{j}}, \quad n \in \mathbb{N} .
$$

Then $\left|f_{n}(z)\right| \leq 2 C_{1}$ for every $z \in \mathbb{D}_{\rho} \backslash \bigcup_{j=1}^{n} \overline{\mathbb{D}}\left(a_{j}, \frac{\rho_{j}}{2}\right)$ and all $n$.

Let $h \in \operatorname{PSH}\left(D_{2} \times \mathbb{C}\right)$ have the property that $h(z, f(z))=-\infty, z \in D_{1}$. The function $s_{n}$ defined on $D_{2} \backslash\left\{a_{1}, \ldots, a_{n}\right\}$ by $s_{n}(z):=h\left(z, f_{n}(z)\right)$ is subharmonic. Let $A_{n}:=\sup _{z \in J} s_{n}(z)$ and let $C_{2}:=\sup _{z \in \overline{\mathbb{D}}_{\rho},|w| \leq 2 C_{1}} h(z, w)$. Then $A_{n} \rightarrow \sup _{z \in J} h(z, f(z))=-\infty$ as $n \rightarrow \infty$.

From the two-constant theorem (see e.g. [8], Theorem 4.3.7) we infer

$$
\frac{C_{2}-s_{n}(0)}{C_{2}-A_{n}} \geq \omega\left(0, J, \mathbb{D}_{\rho} \backslash \bigcup_{j=1}^{n} \overline{\mathbb{D}}\left(a_{j}, \frac{\rho_{j}}{2}\right)\right) \geq \frac{1}{2 n_{0}}, \quad n \in \mathbb{N} .
$$

Letting $n \rightarrow \infty$, we conclude that $h(0, f(0))=s_{n}(0)=-\infty$ and therefore $(0, f(0)) \in$ $\left(\Gamma_{f}\right)_{D_{2} \times \mathbb{C}}^{*}$.

For the proof of Theorem 1.1 we need to know that the function defined by (2.1) is not extendible across the boundary of $D_{1}$. This will be done in the next section.

\section{NON-EXTENDIBLE SUMS}

Without additional conditions on $a_{n}$ and $c_{n}$ a function defined by (2.1) may well extend holomorphically beyond the boundary of $D_{1}$-think of the Lambert-type series $\sum_{n=1}^{\infty} c_{n} \frac{z^{n}}{1-z^{n}}$; cf. [5]. It may even yield 0 on $D_{1}$; cf. 11. We will see that a suitable choice of $a_{n}$ and $c_{n}$ prevents this from happening. We are grateful to Marek Jarnicki and Peter Pflug who suggested the idea of the proof of the next lemma.

Lemma 3.1. Let $D$ be a domain in $\mathbb{C}$. Then there exist a dense subset $A=\left\{a_{n}\right\}_{n=1}^{\infty}$ of $\partial D$ and a sequence $\left\{R_{n}\right\}_{n=1}^{\infty}$ of positive numbers such that for any sequence of complex numbers $\left\{c_{n}\right\}_{n=1}^{\infty}$ with $0<\left|c_{n}\right| \leq R_{n}$ the holomorphic function $f$ given by (2.1) is not holomorphically extendible across $\partial D$.

Proof. Let $B=\left\{b_{n}\right\}_{n=1}^{\infty}$ be a dense subset of $D$ (take e.g. $U=D \cap \mathbb{Q}^{2}$ ). For any $b_{n} \in B$ there exists a point $a \in \partial D$ such that $\operatorname{dist}\left(b_{n}, \partial D\right)=\left|b_{n}-a\right|$. We denote by $a_{n}$ one of them. Set $A=\left\{a_{n}\right\}_{n=1}^{\infty}$. Note that $A$ is a dense subset of $\partial D$. Taking subsequence of $\left\{a_{n}\right\}_{n=1}^{\infty}$ we may assume that $a_{i} \neq a_{j}, i \neq j$.

Fix $n \in \mathbb{N}$. Let $B_{n}=\left\{z \in D: \operatorname{dist}(z, \partial D)=\left|z-a_{n}\right|\right\} \subset D$. Note that $B_{n} \cup\left\{a_{n}\right\}$ is a closed set on the plane and $\widetilde{B}=\bigcup_{n=1}^{\infty} B_{n}$ is dense in $D$ (because $\widetilde{B} \supset B$ ). Moreover, if $z_{0} \in B_{n}$, then the open segment with the ends at the points $z_{0}$ and $a_{n}$ is contained in $B_{n}$. 
For any $j \in \mathbb{N}$ we put $\epsilon_{n j}=\operatorname{dist}\left(a_{j}, B_{n}\right)$. Since $a_{j} \notin B_{n}$ for $j \neq n$, we see that $\epsilon_{n j}>0$ for $j \neq n$.

Put

$$
R_{j}=\frac{\min \left\{\epsilon_{1 j}, \ldots, \epsilon_{(j-1) j}\right\}}{j^{2}}, \quad j \in \mathbb{N} .
$$

For any $n \in \mathbb{N}$ and any $j>n$ we have $R_{j} \leq \frac{\epsilon_{n j}}{j^{2}}$ and therefore

$$
\sum_{j \neq n} \frac{R_{j}}{\left|z-a_{j}\right|} \leq \sum_{j \neq n} \frac{R_{j}}{\epsilon_{n j}}<+\infty, \quad z \in B_{n} .
$$

Take a sequence of complex numbers $\left\{c_{n}\right\}_{n=1}^{\infty}$ with $0<\left|c_{n}\right| \leq R_{n}$. Then for a fixed $n \in \mathbb{N}$ we have

$$
\begin{aligned}
& \liminf _{B_{n} \ni z \rightarrow a_{n}}\left|\left(z-a_{n}\right) f(z)\right| \\
& \quad \geq\left|c_{n}\right|-\lim _{B_{n} \ni z \rightarrow a_{n}}\left|z-a_{n}\right| \cdot \limsup _{B_{n} \ni z \rightarrow a_{n}} \sum_{j \neq n} \frac{\left|c_{j}\right|}{\left|z-a_{j}\right|}=\left|c_{n}\right|>0 .
\end{aligned}
$$

Observe that for any $n \in \mathbb{N}$ the Taylor series at any point of $z_{0} \in B_{n}$ has a radius of convergence equal to $\operatorname{dist}\left(z_{0}, \partial D\right)$ (because of (3.1) and $\left|z_{0}-a_{n}\right|=\operatorname{dist}\left(z_{0}, \partial D\right)$ ). Hence, by Lemma 1.7.5 from [3] we see that $D$ is the domain of existence of $f$.

Proof of Theorem 1.1. If a set $E \subset \Omega$ is complete pluripolar in a domain $\Omega$, then $E_{\Omega}^{*}=E$. By Lemma 3.1 and Theorem 2.1 there exists a holomorphic function $f$ on $D_{1}$ for which $D_{1}$ is a domain of existence and $\left(\Gamma_{f}\right)_{D_{2} \times \mathbb{C}}^{*} \neq \Gamma_{f}$. Hence, $\Gamma_{f}$ is not complete pluripolar in $D_{2} \times \mathbb{C}$.

Theorem 3.2. There exists a sequence $\left\{a_{n}\right\}_{n=1}^{\infty} \subset \mathbb{C} \backslash \overline{\mathbb{D}}$ and a sequence $\left\{c_{n}\right\}_{n=1}^{\infty}$ such that the function $f$ defined by (2.1) is $C^{\infty}$ on $\overline{\mathbb{D}}$ and is nowhere extendible over the boundary of $\mathbb{D}$, while $\Gamma_{f}$ is not complete pluripolar in $\mathbb{C}^{2}$.

Proof. Let $r_{j}=1+1 /(j+1)$. The sequence $a_{n}$ is formed by

$$
a_{2^{j}+k}=r_{j} e^{2 \pi i \frac{k}{2^{j}}}, \quad k=0, \ldots, 2^{j}-1, j=0,1, \ldots
$$

The proof of Theorem 2.1 provides us with a sequence $\left\{R_{n}\right\}$ such that for every sequence $\left\{c_{n}\right\}$ with $\left|c_{n}\right|<R_{n}$ the series (2.1) represents a function on $\mathbb{D}$, the graph of which is not complete pluripolar. Assembling all $a_{n} \in C\left(0, r_{j}\right)$ we find that there exists a sequence $\left\{R_{j}^{\prime}\right\}$ such that for every choice of $0<\varepsilon_{j}<R_{j}^{\prime}$ the function $f_{\varepsilon}$ on $\mathbb{D}$ defined by

$$
f_{\varepsilon}(z)=\sum_{j=0}^{\infty} \frac{\varepsilon_{j}}{r_{j}^{2^{j}}-z^{2^{j}}}
$$

has a graph that is not complete pluripolar.

We observe that independent of the choice of $\varepsilon_{j}$,

$$
\sum_{j=1}^{n} \frac{\varepsilon_{j}}{r_{j}^{2^{j}}-z^{2^{j}}}=\sum_{k=1}^{\infty} d_{n, k} z^{k}
$$

is holomorphic on $\mathbb{D}_{r_{n}}$ with singularities on the boundary. (The $d_{n, k}$ are defined by the equality.) Therefore we have $\lim \sup _{k \rightarrow \infty}\left|d_{n, k}^{1 / k}\right|=1 / r_{n}$. Hence there is a $k_{n}$ such that

$$
\left|d_{n, k_{n}}\right|>r_{n-1}^{-k_{n}}
$$


We will now make an appropriate choice for the $\varepsilon_{j}$ to insure that $f$ cannot be extended over the boundary of $\mathbb{D}$. Along the way we will determine constants $C_{j}$ that are needed for smoothness at the boundary.

Choose $\varepsilon_{0}=R_{0}^{\prime}$. Then

$$
f_{\varepsilon_{0}}(z)=\frac{1}{r_{0}-z}=\sum_{k=1}^{\infty} d_{0, k} z^{k}
$$

with $\lim \sup _{k \rightarrow \infty}\left|d_{0, k}^{1 / k}\right|=1 / r_{0}$; in particular there is a $C_{0}$ such that $\left|d_{0, k}\right|<C_{0}$.

Suppose $\varepsilon_{0}, \ldots, \varepsilon_{n-1}$ and $C_{0}, \ldots, C_{n-1}$ have been chosen in such a way that we have found $k_{0}, \ldots, k_{n-1}$ with

$$
\left|d_{l, k_{j}}\right|>r_{j-1}^{-k_{j}} \text { for } j=1, \ldots, n-1, l=j, \ldots, n-1,
$$

and

$$
\left|d_{j, k}\right|<\frac{C_{l}}{k^{l}} \quad \text { for } l=0, \ldots, n-1, j=0, \ldots, n-1, \quad \text { and all } k .
$$

Then choose

$$
C_{n}>\sup _{k}\left|d_{j, k}\right| k^{n} \quad j=0, \ldots, n-1 .
$$

This is finite because of (3.4). Next choose $\varepsilon_{n}<R_{n}^{\prime}$ so small that

(1) The inequality (3.5) holds for $l=0, \ldots, n$ and $j=0, \ldots, n$. This is possible because of (3.4).

$$
\left|d_{n, k_{j}}\right|>r_{j-1}^{-k_{j}} \text { for } j=1, \ldots, n-1,
$$

which is again possible because of (3.4).

Having chosen $\varepsilon_{n}$, we can by (3.3) choose $k_{n}$ so large that $\left|d_{n, k_{n}}\right|>r_{n-1}^{-k_{n}}$.

Observe that the coefficients $d_{n, k}$ converge to the coefficients $d_{k}$ of the power series expansion of $f_{\varepsilon}$ as $n \rightarrow \infty$. From (3.4) we see that $\left|d_{k_{j}}\right|>r_{j-1}^{-k_{j}}$ so that the radius of convergence of the power series of $f_{\varepsilon}$ is at most 1 , and since $f_{\varepsilon}$ is holomorphic on $\mathbb{D}$, it equals 1 . So $f_{\varepsilon}$ has a singular point $b$ on $C(0,1)$. We split $f_{\varepsilon}$ as

$$
f_{\varepsilon}=f_{1}+f_{2}=\left(\sum_{j=0}^{n-1}+\sum_{j=n}^{\infty}\right) \frac{\varepsilon_{j}}{r_{j}^{2^{j}}-z^{2^{j}}} .
$$

Then $f_{1}$ is holomorphic in a neighborhood of the closed unit disc and $f_{2}$ has at least one singular point on $C(0,1)$, but $f_{2}$ is invariant under rotation over a $2^{n}$-th root of unity, which implies that there is a singularity in each arc of length $>2 \pi / 2^{n}$. Therefore, $f$ can nowhere be extended analytically over $C(0,1)$.

Next we show that $f$ is smooth up to the boundary of $\mathbb{D}$. We have to show that there exist constants $C_{l}>0$ such that for every $l$

$$
\left|d_{k}\right| \leq \frac{C_{l}}{k^{l}}, \quad \text { for all } k
$$

but this follows from (3.5). 
Remark 3.3. Let $D$ be a domain in $\mathbb{C}$ and let $A$ be a closed polar subset of $D$. Using methods presented in this paper and in [10], the authors give in [2] a complete characterization of the holomorphic functions $f$ on $D \backslash A$ such that $\Gamma_{f}=\{(z, f(z))$ : $z \in D \backslash A\}$ is complete pluripolar in $D \times \mathbb{C}$.

\section{REFERENCES}

1. L. Brown, A. Shields \& K. Zeller, On absolutely convergent exponential sums, Trans. Am. Soc., 96 (1960), 162-183. MR 26:332

2. A. Edigarian \& J. Wiegerinck, The pluripolar hull of the graph of a holomorphic function with polar singularities, Indiana Univ. Math. J., to appear.

3. M. Jarnicki \& P. Pflug, Extension of holomorphic functions, De Gruyter Expositions in Mathematics 34, 2000. MR 2001k:32017

4. M. Klimek, Pluripotential Theory, London Math. Soc. Monographs, 6, Clarendon Press, 1991. MR 93h:32021

5. K. Knopp, Theorie und Anwendung der unendlichen Reihen, Grundl. Math. Wiss. 2, 5-th ed. Springer Verlag, 1964. MR 32:1473

6. N. Levenberg, G. Martin \& E.A. Poletsky, Analytic disks and pluripolar sets, Indiana Univ. Math. J., 41 (1992), 515-532. MR 93h:46075

7. N. Levenberg \& E.A. Poletsky, Pluripolar hulls, Michigan Math. J., 46 (1999), 151-162. MR 2000b:32063

8. Th. Ransford, Potential Theory in the Complex Plane, Cambridge University Press, 1994. MR 96e:31001

9. J. Wiegerinck, The pluripolar hull of $\left\{w=e^{-\frac{1}{z}}\right\}$, Ark. Mat., 38 (2000), 201-208. MR 2001b:32070

10. J. Wiegerinck, Graphs of holomorphic functions with isolated singularities are complete pluripolar, Michigan Math. J., 47 (2000), 191-197. MR 2001b:32071

11. J. Wiegerinck, Pluripolar sets: hulls and completeness. In: G. Raby \& F. Symesak (eds.) Actes des renctres d'analyse complexe, Atlantiques, 2000.

Institute of Mathematics, Jagiellonian University, Reymonta 4/526, 30-059 Kraków, POLAND

E-mail address: edigaria@im.uj.edu.pl

Faculty of Mathematics, University of Amsterdam, Plantage Muidergracht 24, 1018 TV, Amsterdam, The Netherlands

E-mail address: janwieg@science.uva.nl 